\title{
Temperature-dependent transformation of the magnetic excitation spectrum on approaching superconductivity in $\mathrm{Fe}_{1-x}(\mathrm{Ni} / \mathrm{Cu})_{x} \mathrm{Te}_{0.5} \mathrm{Se}_{0.5}$
}

\author{
Zhijun Xu,, , Jinsheng Wen, ${ }^{1,2,3, \text { 冈 }}$ Yang Zhao, ${ }^{4,5}$ Masaaki Matsuda, ${ }^{6}$ Wei Ku, ${ }^{1}$ Xuerong \\ Liu, ${ }^{1}$ Genda Gu, ${ }^{1}$ D.-H. Lee, ${ }^{2,3}$ R. J. Birgeneau, ${ }^{2,3}$ J. M. Tranquada, ${ }^{1}$ and Guangyong $\mathrm{Xu}^{1}$ \\ ${ }^{1}$ Condensed Matter Physics and Materials Science Department, \\ Brookhaven National Laboratory, Upton, New York 11973, USA \\ ${ }^{2}$ Physics Department, University of California, Berkeley, CA 94720, USA \\ ${ }^{3}$ Materials Science Division, Lawrence Berkeley National Laboratory, Berkeley, CA, 94720, USA \\ ${ }^{4}$ NIST Center for Neutron Research, National Institute of Standards and Technology, Gaithersburg, Maryland 20899, USA \\ ${ }^{5}$ Department of Materials Science and Engineering, University of Maryland, College Park, Maryland, 20742, USA \\ ${ }^{6}$ Quantum Condensed Matter Division, Oak Ridge National Laboratory, Oak Ridge, TN, 37831, USA
}

(Dated: November 8, 2018)

\begin{abstract}
Spin excitations are one of the top candidates for mediating electron pairing in unconventional superconductors. Their coupling to superconductivity is evident in a large number of systems, by the observation of an abrupt redistribution of magnetic spectral weight at the superconducting transition temperature, $T_{c}$, for energies comparable to the superconducting gap. Here we report inelastic neutron scattering measurements on Fe-based superconductors, $\mathrm{Fe}_{1-x}(\mathrm{Ni} / \mathrm{Cu})_{x} \mathrm{Te}_{0.5} \mathrm{Se}_{0.5}$, that emphasize an additional signature. The overall shape of the low energy magnetic dispersion changes from two incommensurate vertical columns at $T \gg T_{c}$ to a distinctly different U-shaped dispersion at low temperature. Importantly, this spectral reconstruction is apparent for temperature up to $\sim 3 T_{c}$. If the magnetic excitations are involved in the pairing mechanism, their surprising modification on the approach to $T_{c}$ demonstrates that strong interactions are involved.
\end{abstract}

In weak-coupling models of magnetically-mediated superconductivity, magnons essentially replace phonons as the pairing bosons [1]. By assumption, the interaction between the electrons and bosons is not strong enough to modify the bosonic excitation spectrum. In conventional systems, superconductivity does modify the self-energy of the phonons, but there is no significant change in the phonon dispersion [2]. In many unconventional superconductors, including high- $T_{c}$ cuprates [3-6], heavy Fermion superconductors [7, 8], and the recently discovered Fe-based superconductors [9-11], one observes, on cooling below $T_{c}$, the gapping of low-energy spin fluctuations and a shift of spectral weight to a "resonance" peak. Empirically, the magnetic spectrum found above and below $T_{c}$ tends to be qualitatively the same.

Here we study the low-energy spin fluctuations in singlecrystal samples of the superconductor $\mathrm{FeTe}_{0.5} \mathrm{Se}_{0.5}$ (the "1:1" system, $T_{c}=14 \mathrm{~K}$ ) as we perturb the system by making partial substitutions for $\mathrm{Fe}$. Substituting $2 \%$ and $4 \%$ of Ni reduces $T_{c}$ to $12 \mathrm{~K}$ and $8 \mathrm{~K}$, respectively, while $10 \%$ of $\mathrm{Cu}$ results in an absence of superconductivity, as shown in Fig. 1.(a). Our inelastic neutron scattering measurements show that low energy ( $\hbar \omega \lesssim 12 \mathrm{meV}$ ) magnetic excitations transform from having two peaks clearly away from the antiferromagnetic (AF) wave-vector at high temperature in the normal state, to having a broad maximum near the AF wave-vector at low temperature in the superconducting phase. This drastic change on the magnetic dispersion between the superconducting and nonsuperconducting phases suggests that strong correlations between electrons have to be taken into account when the magnetic and electronic properties of the " $1: 1$ " system are considered.

Single crystals of $\mathrm{Fe}_{1-x}(\mathrm{Ni} / \mathrm{Cu})_{x} \mathrm{Te}_{0.5} \mathrm{Se}_{0.5}$ were grown by a unidirectional solidification method [12] at Brookhaven Na- tional Laboratory. The lattice constants are $a=b=3.81 \AA$, and $c=6.02 \AA$, using the two-Fe unit cell. For convenience, we label these samples as $\mathrm{NiO2,} \mathrm{Ni04,} \mathrm{and} \mathrm{Cu} 10$, according to the amount of $\mathrm{Ni} / \mathrm{Cu}$ doping on the $\mathrm{Fe}$ site. The neutron scattering experiments on the two Ni02 and Ni04 samples were carried out on the BT7 triple-axis-spectrometer at the NIST Center for Neutron Research, using beam collimations of open-50'-S-50'-240', a fixed final energy of $14.7 \mathrm{meV}$ and two pyrolytic graphite filters after the sample. The $\mathrm{Cu} 10$ sample was measured on the HB1 triple-axis-spectrometer at the High Flux Isotope Reactor, Oak Ridge National Laboratory. with beam collimations of $48^{\prime}-40^{\prime}-\mathrm{S}-60^{\prime}-240^{\prime}$, fixed final energy of $13.5 \mathrm{meV}$, and two pyrolytic graphite filters after the sample. No static order around $(0.5,0,0.5)$ was found in any of the three samples. The inelastic scattering experiments were all performed in the $(H K 0)$ zone, so that the scattering plane is defined by the [100] and [010] wave-vectors. All data have been normalized into absolute units of $\mu_{B}^{2} e V^{-1} / \mathrm{Fe}$ by incoherent elastic scattering intensities from the samples. Xray diffraction measurements of lattice parameters were performed at beamline X22B of the National Synchrotron Light Source, Brookhaven National Laboratory.

We are interested in the magnetic excitations near the $\mathrm{AF}$ wave-vector $\mathbf{Q}_{\mathrm{AF}}=(0.5,0.5,0)$. Figure 1 (c)-(e) shows the measured inelastic neutron scattering intensity as a function of energy obtained at $T=2.8 \mathrm{~K}$ and $15 \mathrm{~K}$ for all three samples. It has been established in previous studies [13-16] that the unperturbed superconductor has a magnetic resonance peak at $E_{r} \sim 7 \mathrm{meV}$. Here we see that $E_{r}$ decreases to $\sim 6$ and $5 \mathrm{meV}$ in the Ni02 and N04 samples, respectively, while there is no observable resonance in the nonsuperconducting $\mathrm{Cu} 10$. One can also see a spin gap of about $3 \mathrm{meV}$ in $\mathrm{Ni02}$, but the gap is more difficult to resolve for $\mathrm{Ni} 04$. 
Things get more interesting when we look at the wavevector $(\mathbf{q})$ dependence of the magnetic scattering. It has been established in previous studies [13, 14, 17] of superconducting $\mathrm{FeTe}_{1-x} \mathrm{Se}_{x}$ that the magnetic excitations disperse from $\mathbf{Q}_{\mathrm{AF}}$ only in the transverse direction, along $[1,-1,0]$. Figure 2 shows scans along this direction for the Ni04 sample at a series of energies, illustrating the variation of the $\mathbf{q}$ dependence as the temperature changes from $2.8 \mathrm{~K}\left(\ll T_{c}\right)$ to $15 \mathrm{~K}$ $\left(\gtrsim T_{c}\right)$ and then up to $100 \mathrm{~K}\left(T \gg T_{c}\right)$. The variations are minor at the higher energies, as in Fig. 22(e)-(f), but become dramatic for $E \sim E_{r} \approx 5 \mathrm{meV}$, as in Fig. 22(a)-(c). The change from $T \ll T_{c}$ to $T \gtrsim T_{c}$ is simply the standard resonance behavior. The feature that we wish to emphasize is the change from a single commensurate peak at $T \gtrsim T_{c}$ to a pair of wellresolved incommensurate peaks at $T \gg T_{c}$. This change cannot be confused with a temperature-dependent change in peak width.

The same data are presented again, slightly cleaned up and in a different format, in Fig. 3 (a)-(c). The lower-temperature data exhibit a U-shaped dispersion, with the bottom of the U at $\sim E_{r}$. Except for the change in the resonant peak, the basic shape of the dispersion does not really change on crossing $T_{c}$. In contrast, the dispersion at $100 \mathrm{~K}$ is qualitatively different: it looks like the legs of a pair of trousers. It also looks very similar to the low-temperature dispersion of the
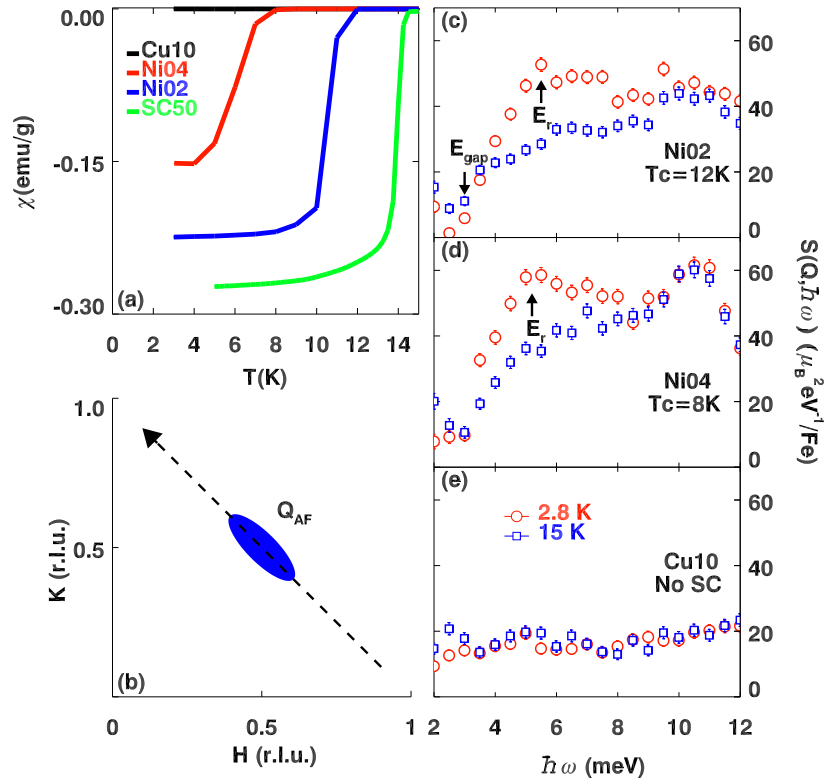

FIG. 1. (Color online) Magnetic susceptibility and inelastic neutron scattering measurements performed on the $\mathrm{NiO2}, \mathrm{Ni04}, \mathrm{Cu} 10$, and SC50 ( $\left.\mathrm{FeTe}_{0.5} \mathrm{Se}_{0.5}\right)$ samples. (a) Magnetic susceptibility measurements, and (c), (d), (e) magnetic neutron scattering intensity measured at $\mathbf{Q}_{\mathrm{AF}}$ with $T=2.8 \mathrm{~K}$ (red circles) and $15 \mathrm{~K}$ (blue squares). The error bars represent the square root of the number of counts. Fitted background obtained from constant-energy scans has been subtracted from all data sets. The (HK0) scattering plane is plotted in (b) while the dashed line denotes the direction for the Q-scans shown in Fig. 2

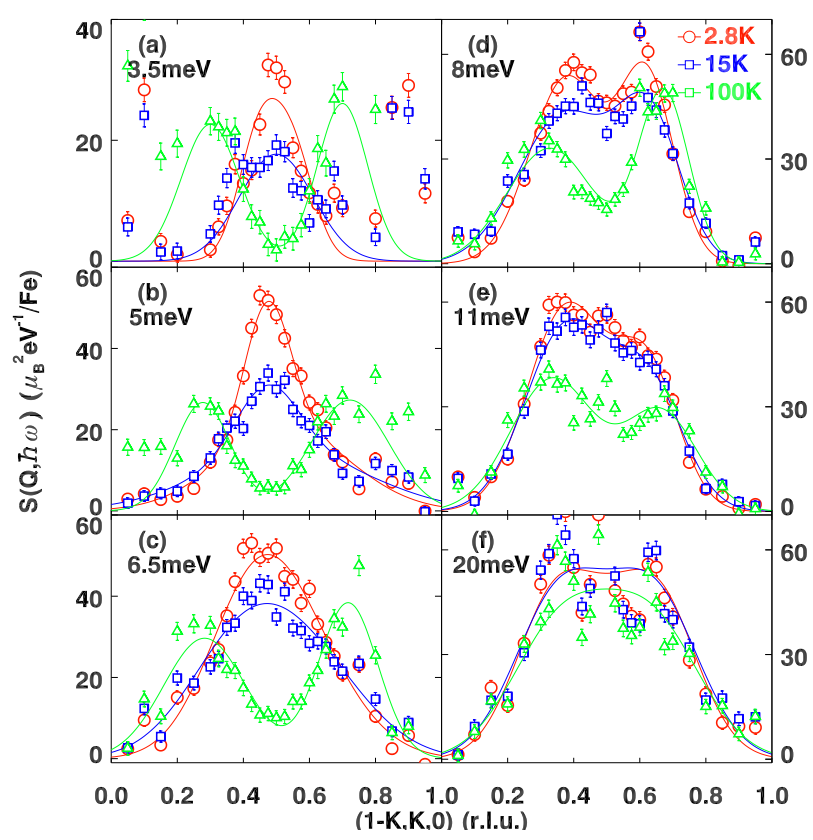

FIG. 2. (Color online) Wave-vector dependence of the magnetic scattering intensity along the transverse direction through $\mathbf{Q}_{\mathrm{AF}}$ [see Fig. 1 (b)] for the Ni04 sample at $T=2.8 \mathrm{~K}$ (red circles), $15 \mathrm{~K}$ (blue squares), and $100 \mathrm{~K}$ (green triangles), obtained at excitation energies (a) $3.5 \mathrm{meV}$, (b) $5 \mathrm{meV}$, (c) $6.5 \mathrm{meV}$, (d) $8 \mathrm{meV}$, (e) $11 \mathrm{meV}$, (f) $20 \mathrm{meV}$ [which was measured in a higher zone, near $\mathbf{Q}=(1.5,0.5,0)]$. Solid lines are guides to the eye.

non-superconducting Cu10 sample shown in Fig. 3(d).

There is clearly a major change in the low-energy portion of the dispersion between 15 and $100 \mathrm{~K}$, but how does it change between those temperatures? This is illustrated in Fig. 4, Focusing in particular on the results for the Ni04 sample, in Fig. 4(e) we see that the crossover is continuous in temperature, but with a reasonably defined mid-point at $30 \pm 10 \mathrm{~K}$. For Ni02, the midpoint may be closer to $40 \mathrm{~K}$. In both cases, the crossover occurs at temperatures of order $3 T_{c}$. We previously observed [16] hints of this temperature dependent modification of the dispersion in superconducting $\mathrm{FeTe}_{0.35} \mathrm{Se}_{0.65}$; however, the high-temperature incommensurability was not as large nor as well resolved as for the $\mathrm{Ni}$ - and $\mathrm{Cu}$-doped samples [see Fig. 囵(e)].

It is possible to see the incommensurate columns of magnetic scattering even at low temperature when the superconductivity is suppressed, as shown for the Cu10 sample in Fig. 3(d). A similar low-temperature spectrum has been observed previously in non-bulk-superconducting "1:1" samples such as $\mathrm{Fe}_{1.04} \mathrm{Te}_{0.73} \mathrm{Se}_{0.27}$ [17] and $\mathrm{Fe}_{1.10} \mathrm{Te}_{0.75} \mathrm{Se}_{0.25}$ [18].

There is an evident pattern that superconducting 1:1 samples have commensurate or almost commensurate magnetic excitations at the resonance energy, while nonsuperconducting samples have incommensurate excitations. Our results for the Ni-doped samples show that it is possible for a sample to transform from the incommensurate phase at 
(a)
$2.8 \mathrm{~K}$

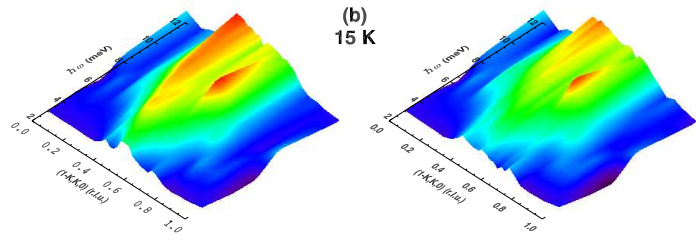

(c)
$100 \mathrm{~K}$

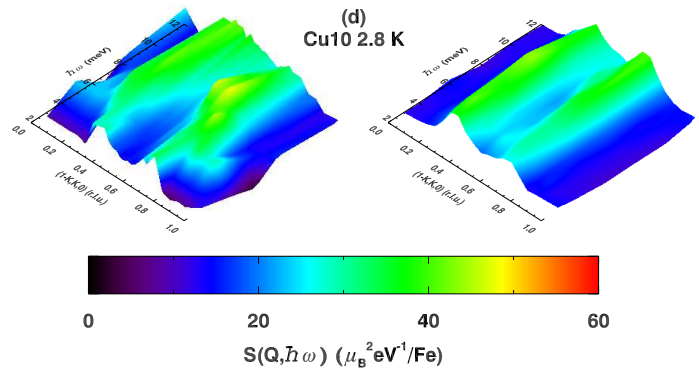

FIG. 3. (Color online) Magnetic scattering intensity plotted for the Ni04 sample in energy-momentum space at (a) $2.8 \mathrm{~K}$, (b) $15 \mathrm{~K}$, and (c) $100 \mathrm{~K}$. Results for the $\mathrm{Cu} 10$ sample measured at $2.8 \mathrm{~K}$ are plotted in (d). The data have been smoothed, and non-magnetic sharp spurious signals [see Fig. [2]a)] have been removed for better visual effects.

high temperature to the low-energy-commensurate phase on cooling. The commensurability appears at the energy scale of the resonance energy at a temperature of $\sim 3 T_{c}$. One possible explanation of this incommensurate-to-commensurate transformation may be related to the strong orbital correlations in this system. In the iron-based superconductors, it has been proposed that there are competing electronic instabilities similar to those in the cuprates [19, 20]. In addition to antiferromagnetism and superconductivity, the material also has a propensity toward $x z / y z$ orbital ordering. Such ordering can modify the shape of the Fermi surface (e.g., enlarging one electron Fermi pocket while shrinking the other), leading to enhancement of the commensurate magnetic scattering between the hole and the electron pockets [20]. Even if longrange orbital ordering does not set in upon cooling, the system may still have regions of locally ordered or slowly fluctuating orbital correlations. It is possible that the crossover we observe at $\sim 3 T_{c}$ reflects such an orbital ordering "transition" in the presence of disorder. An additional experimental support of this scenario is given by $\mathrm{x}$-ray scattering measurements on $\mathrm{Fe}_{1+y} \mathrm{Se}_{0.57} \mathrm{Te}_{0.43}$ [21] where it was shown that a weak lattice distortion, reflecting the breaking of 90 degree rotation symmetry, sets in around $40 \mathrm{~K}$, similar to the crossover temperature observed in our experiment. In our samples, we did not see such a lattice distortion. Nevertheless, the temperature dependence of the lattice parameters (Fig. 5) obtained from the $\mathrm{Ni04}$ sample indicate an anomalous in-plane expansion below $60 \mathrm{~K}$, while $c$ decreases monotonically with cooling.

This temperature-dependent transformation of the mag-

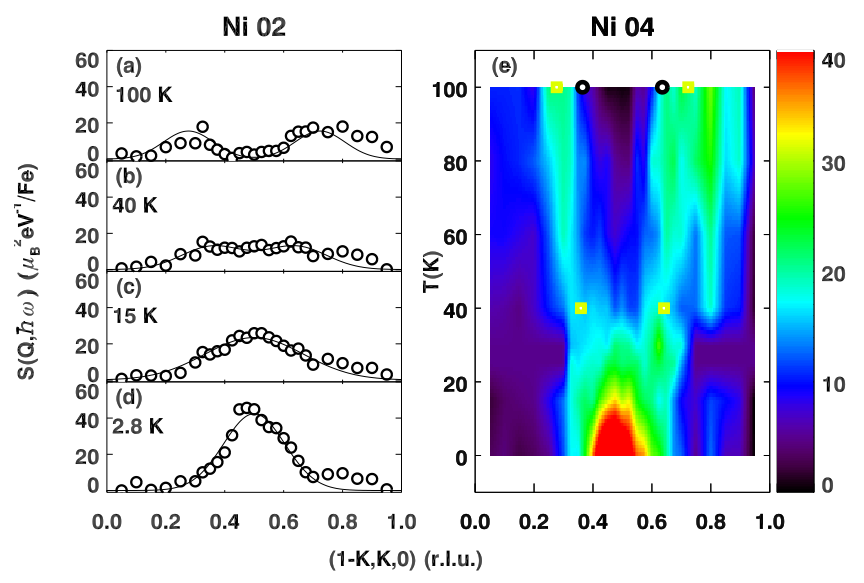

FIG. 4. (Color online) Thermal evolution of the magnetic scattering at $\hbar \omega=5 \mathrm{meV}$. The data are measured through $\mathbf{Q}_{\mathrm{AF}}$ along the transverse direction for the Ni02 sample at (a) $100 \mathrm{~K}$, (b) $40 \mathrm{~K}$, (c) $15 \mathrm{~K}$, (d) $2.8 \mathrm{~K}$, and (e) for the Ni04 sample plotted as an intensity contour map in temperature-wave-vector space. The data have been smoothed. The yellow and black symbols in (e) denote the corresponding peak positions for the Ni02 sample (yellow squares) and for a superconducting $\mathrm{Fe}_{1+\delta} \mathrm{Te}_{0.35} \mathrm{Se}_{0.65}$ sample [16].

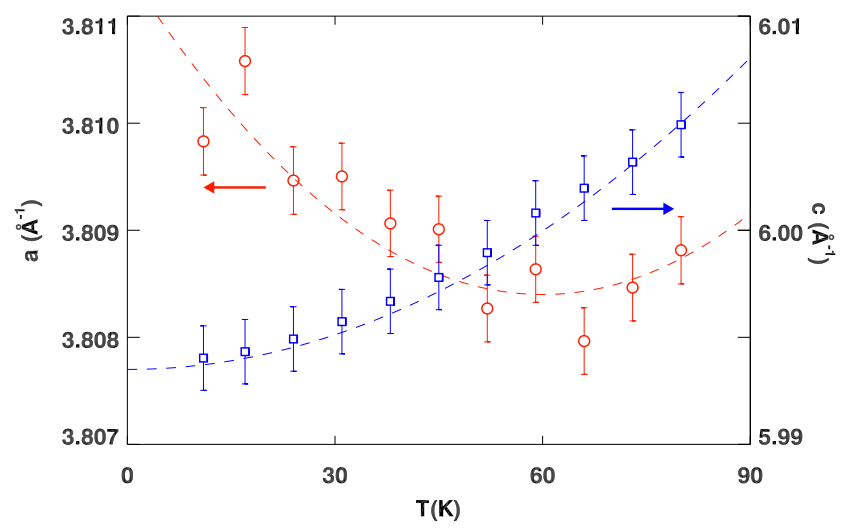

FIG. 5. (Color online) Lattice parameters $a$ (red circle) and $c$ (blue squares) measured on the Ni04 sample.

netic spectrum is unusual among unconventional superconductors. For example, in superconducting $\mathrm{YBa}_{2} \mathrm{Cu}_{3} \mathrm{O}_{6+x}$ systems [3-5], the spin resonance develops at commensurate wave-vectors below $\mathrm{T}_{c}$, but the "hour-glass" shaped dispersion with a commensurate saddle point is still compatible with the high temperature spectrum, within the increased $\mathrm{q}$ widths. In superconducting $\mathrm{La}_{2-x} \mathrm{Sr}_{x} \mathrm{CuO}_{4}$ the spin resonance occurs at lower energies where the spin fluctuations are incommensurate [22, 23], both in the normal and superconducting phases. Returning to the analogy with electronphonon coupling, strong interactions can lead to a modification of the spectrum through a structural phase transition, as 
occurs [24] in $\mathrm{Nb}_{3} \mathrm{Sn}$ at a temperature above the superconducting $T_{c}$. In the present case, strong interactions appear necessary to cause the transformation from incommensurate to commensurate magnetic excitations. It is therefore reasonable to expect that measurements on the electronic structures in the system may also provide hints of this transformation in the same temperature range. We note that a related precursor evolution of the charge response is apparent in the optical conductivity of 1:1 superconductor [25]. It may also be worth noting that a thermally-induced enhancement of magnetic moments has been identified in non-superconducting $\mathrm{Fe}_{1.1} \mathrm{Te}[26$ ] a similar temperature scale.

Commensurate excitations are needed for the spinfluctuation mechanism of electron pairing considered by a number of authors [19, 27-30]. In such a scenario, the momentum of the repulsive spin excitations couples the nearlynested hole and electron pockets, and in turn allows a superconducting gap to develop on both sets of pockets, though with opposite phases. Obviously, losing the commensuration of the spin excitations would seriously impair the development of superconductivity in this kind of weak coupling scenario. On cooling, do the electronic and magnetic correlations adjust themselves to enable the spin-fluctuation mechanism? If so, what are the energetic tradeoffs associated with this transformation? And can interactions strong enough to achieve this transformation lead to effectively the same pairing mechanism as the one identified from a weak-coupling approach? We hope that these questions will be addressed by future investigations.

We thank Igor Zaliznyak for useful discussions. Work at BNL is supported by the Office of Basic Energy Sciences, U.S. Department of Energy under contract No. DE-AC0298CH10886. Work at Berkeley is supported by the same office through contract No. DE-AC02-05CH11231. The research at ORNL was sponsored by the Scientific User Facilities Division, Office of Basic Energy Sciences, U. S. DOE.

* ZJX and JSW contributed equaly to this work.

[1] D. J. Scalapino, Physica C 470, S1 (2010).

[2] F. Weber and L. Pintschovius, Phys. Rev. B 82, 024509 (2010)

[3] J. Rossatmignod, L. P. Regnault, C. Vettier, P. Bourges, P. Burlet, J. Bossy, J. Y. Henry, and G. Lapertot, Physica C 185, 86 (1991).

[4] P. Bourges, L. P. Ragnault, Y. Sidis, and C. Vettier, Phys. Rev. B 53, 876 (1996).

[5] P. C. Dai, H. A. Mook, G. Aeppli, S. M. Hayden, and F. Dogan, Nature 406, 965 (2000).

[6] H. F. Fong, P. Bourges, Y. Sidis, L. P. Ragnault, A. Ivanov, G. D. Gu, N. Koshizuka, and B. Keimer, Nature 398, 588 (1999).

[7] N. K. Sato, N. Aso, K. Miyake, R. Shiina, P. Thalmeier, G. Varelogiannis, C. Geibel, F. Steglich, P. Fulde, and T. Komatsubara, Nature 410, 340 (2001).
[8] C. Stock, C. Broholm, J. Hudis, H. J. Kang, and C. Petrovic, Physical Review Letters 100, 087001 (2008).

[9] A. D. Christianson, E. A. Goremychkin, R. Osborn, S. Rosenkranz, M. D. Lumsden, C. D. Malliakas, I. S. Todorov, H. Claus, D. Y. Chung, M. G. Kanatzidis, R. I. Bewley, and T. Guidi, Nature 456, 930 (2008).

[10] S. Chi, A. Schneidewind, J. Zhao, L. W. Harriger, L. Li, Y. Luo, G. Cao, Z. A. Xu, M. Loewenhaupt, J. Hu, and P. Dai, Phys. Rev. Lett. 102, 107006 (2009).

[11] Y. Qiu, W. Bao, Y. Zhao, C. Broholm, V. Stanev, Z. Tesanovic, Y. C. Gasparovic, S. Chang, J. Hu, B. Qian, M. Fang, and Z. Mao, Phys. Rev. Lett. 103, 067008 (2009).

[12] J. Wen, G. Xu, G. Gu, J. M. Tranquada, and R. J. Birgeneau, Rep. Prog. Phys. 74, 124503 (2011).

[13] D. N. Argyriou, A. Hiess, A. Akbari, I. Eremin, M. M. Korshunov, J. Hu, B. Qian, Z. Mao, Y. Qiu, C. Broholm, and W. Bao, Phys. Rev. B 81, 220503 (2010).

[14] S. H. Lee, G. Xu, W. Ku, J. S. Wen, C. C. Lee, N. Katayama, Z. J. Xu, S. Ji, Z. W. Lin, G. D. Gu, H. B. Yang, P. D. Johnson, Z. H. Pan, T. Valla, M. Fujita, T. J. Sato, S. Chang, K. Yamada, and J. M. Tranquada, Phys. Rev. B 81, 220502 (2010).

[15] S. Li, C. Zhang, M. Wang, H.-q. Luo, X. Lu, E. Faulhaber, A. Schneidewind, P. Link, J. Hu, T. Xiang, and P. Dai, Phys. Rev. Lett. 105, 157002 (2010).

[16] Z. J. Xu, J. S. Wen, G. Y. Xu, S. X. Chi, W. Ku, G. D. Gu, and J. M. Tranquada, Physical Review B 84, 052506 (2011).

[17] M. D. Lumsden, A. D. Christianson, E. A. Goremychkin, S. E. Nagler, H. A. Mook, M. B. Stone, D. L. Abernathy, T. Guidi, G. J. MacDougall, C. de la Cruz, A. S. Sefat, M. A. McGuire, B. C. Sales, and D. Mandrus, Nat. Phys. 6, 182 (2010).

[18] P. Babkevich, M. Bendele, A. T. Boothroyd, K. Conder, S. N. Gvasaliya, R. Khasanov, E. Pomjakushina, and B. Roessli, J. Physics-Condensed Matter 22, 142202 (2010).

[19] F. Wang and D.-H. Lee, Science 332, 200 (2011).

[20] H. Zhai, F. Wang, and D.-H. Lee, Phys. Rev. B 80, 064517 (2009).

[21] N. C. Gresty, Y. Takabayashi, A. Y. Ganin, M. T. McDonald, J. B. Claridge, D. Giap, Y. Muzuguchi, Y. Takano, T. Kagayama, Y. Ohishi, M. Takata, M. J. Rosseinsky, S. Margadonna, and K. Prassides, J. Am. Chem. Soc 131, 16944 (2009).

[22] N. B. Christensen, D. F. McMorrow, H. M. Rønnow, B. Lake, S. M. Hayden, G. Aeppli, T. G. Perring, M. Mangkorntong, M. Nohara, and H. Takagi, Phys. Rev. Lett. 93, 147002 (2004)

[23] J. M. Tranquada, C. H. Lee, K. Yamada, Y. S. Lee, L. P. Regnault, and H. M. Rønnow, Phys. Rev. B 69, 174507 (2004)

[24] G. Shirane and J. D. Axe, Phys. Rev. B 4, 2957 (1971)

[25] C. C. Homes, A. Akrap, J. S. Wen, Z. J. Xu, Z. W. Lin, Q. Li, and G. D. Gu, Phys. Rev. B 81, 180508 (2010).

[26] I. A. Zaliznyak, Z. Xu, J. M. Tranquada, G. Gu, A. M. Tsvelik, and M. B. Stone, Phys. Rev. Lett. 107, 216403 (2011).

[27] T. A. Maier, S. Graser, D. J. Scalapino, and P. Hirschfeld, Phys. Rev. B 79, 134520 (2009).

[28] H. Ikeda, R. Arita, and J. Kunescaron, Phys. Rev. B 81, 054502 (2010).

[29] A. V. Chubukov, M. G. Vavilov, and A. B. Vorontsov, Phys. Rev. B 80, 140515 (2009).

[30] V. Cvetkovic and Z. Tesanovic, Euro. Phys. Lett. 85, 37002 (2009). 